\title{
LAS HIPÓTESIS DE EDUARDO BOSCÁ SOBRE LA ALIMEN- TACIÓN DEL MEGATERIO Y EL MONTAJE DEL EJEMPLAR DE LA COLECCIÓN RODRIGO BOTET (1902-1928)
}

\author{
M. ${ }^{a}$ Amparo SALINAS JAQUES \\ Apartado de Correos n. ${ }^{\text {40. }} 46080$ Valencia. España. jaques@vodafone.es
}

Salinas Jaques, M. A. 2005. Las hipótesis de Eduardo Boscá sobre la alimentación del megaterio y el montaje del ejemplar de la Colección Rodrigo Botet (1902-1928). [The Eduardo Bosca's hypothesis about the megatherium's feeding and the assembly of the Rodrigo Botet Collection's specimen (1902-1928).] Revista Española de Paleontología, N.E. X, 111-118. ISSN 0213-6937.

\begin{abstract}
The Rodrigo Botet's Collection is composed of fossil mammalians of Argentinian Pleistocene. It is a singular collection in this matter, and probably unique in the world from a historical point of view. It was imported to Valencia (Spain) in 1889, and today is stored at the "Museo Municipal de Ciencias Naturales" (a Natural Sciences Museum, depending on the City Hall of Valencia). Here we will describe the process of assembly of the most popular skeleton from this collection, a very complete specimen of Megatherium americanum Cuvier, 1796, found at the Samborombón River's basin. Also, the opinion of Eduardo Boscá on the way of life of megatheriums is discused. His thoughts and ideas were the reason for a delay in the assembly of this skeleton for more than 25 years and so, when Eduardo Boscá died in 1924, the skeleton remained unassembled. Finally, in 1928, Francisco Beltrán Bigorra finished that work, under a very different conception.
\end{abstract}

Keywords: Rodrigo Botet's Collection, Megatherium americanum, Eduardo Boscá Casanoves, Francisco Beltrán Bigorra, Paleobiology, History of Science.

\section{RESUMEN}

La colección paleontológica J. Rodrigo Botet, formada por restos de mamíferos del Pleistoceno Argentino, es una colección singular en su género y, desde el punto de vista histórico, probablemente única en el mundo. Importada a Valencia (España) en 1889, hoy se encuentra depositada en el Museo Municipal de Ciencias Naturales de Valencia. El montaje del ejemplar más popular de la colección, un individuo muy completo de Megatherium americanum Cuvier, 1796, procedente de la cuenca del Río Samborombón, es el objeto de este artículo. Se informa de los estudios acerca de su modo de vida, llevados a cabo por Eduardo Boscá, y se destaca la influencia determinante de sus criterios en la demora del ensamblaje del ejemplar por más de 25 años. Eduardo Boscá murió en 1924, y fue su sucesor en la dirección del Museo, Francisco Beltrán Bigorra, quien, bajo criterios muy diferentes, culminó el montaje del esqueleto en 1928.

Palabras clave: Colección Rodrigo Botet, Megatherium americanum, Eduardo Boscá Casanoves, Francisco Beltrán Bigorra, Paleobiología, Historia de la Ciencia.

\section{INTRODUCCIÓN}

En la colección paleontológica J. Rodrigo Botet se encuentran representadas al menos trece familias pertenecientes a los órdenes -siguiendo a Alberdi et al. (1995)Carnivora, Cingulata, Tardigrada, Rodentia, Litopterna, Notoungulata, Proboscidea, Perissodactyla y Artiodactyla (Salinas Jaques, 2001). Fue recolectada entre 1880 y 1888 por Enrique de Carles, durante sus expediciones a los depósitos aluviales de la cuenca del Río de la Plata, y comprada a éste por el ingeniero José Rodrigo Botet (Manises, 1842-Madrid, 1915) con el fin de regalarla a Valencia (Boscá Casanoves 1899, 1923; Salinas Jaques, 2001), ciudad donde permanece, desde 1889, bajo la tutela de su Ayuntamiento.

Durante las investigaciones para la reconstrucción de la documentación museológica de esta colección, en lo concerniente al ejemplar objeto de este artículo se observaron 
profundas discrepancias entre las previsiones para su montaje recogidas en documentos de archivo y publicaciones sobre la colección, y lo que se desprendía del análisis de la iconografía histórica del ejemplar. Pese a que el esqueleto se hallaba dispuesto para ser armado ya en 1902, el estudio iconográfico revelaba que el montaje no se había completado hasta 1928. Por otra parte, resultaba extraño que el ejemplar más conspicuo de la colección jamás fuera objeto de una publicación monográfica por parte de Eduardo Boscá (Valencia, 1843-1924), responsable de la colección desde 1891 y primer director del Museo Paleontológico (1900-1924).

$\mathrm{Al}$ extender la investigación a los manuscritos de trabajo del naturalista, conservados de forma fragmenta- ria e inconexa en el Archivo del Museo Paleontológico Rodrigo Botet (AMPRB), y sobre todo, tras el hallazgo de un manuscrito de estudio del ejemplar - en este caso completo - incorporado a un expediente administrativo custodiado en el Archivo Municipal de Valencia (AMV), se pudo reconstruir detalladamente la historia del montaje del megaterio, y determinar las razones que promovieron la demora en su culminación, a saber: el interés de Boscá por montarlo en una actitud que evocara lo más fielmente posible su modo de vida, cuya alimentación él consideraba insectívora, y sobre todo su oposición activa a la permanencia de la colección en el Almudín, antigua alhóndiga en estado de ruina ya en 1874 (Aguilar, 1996).

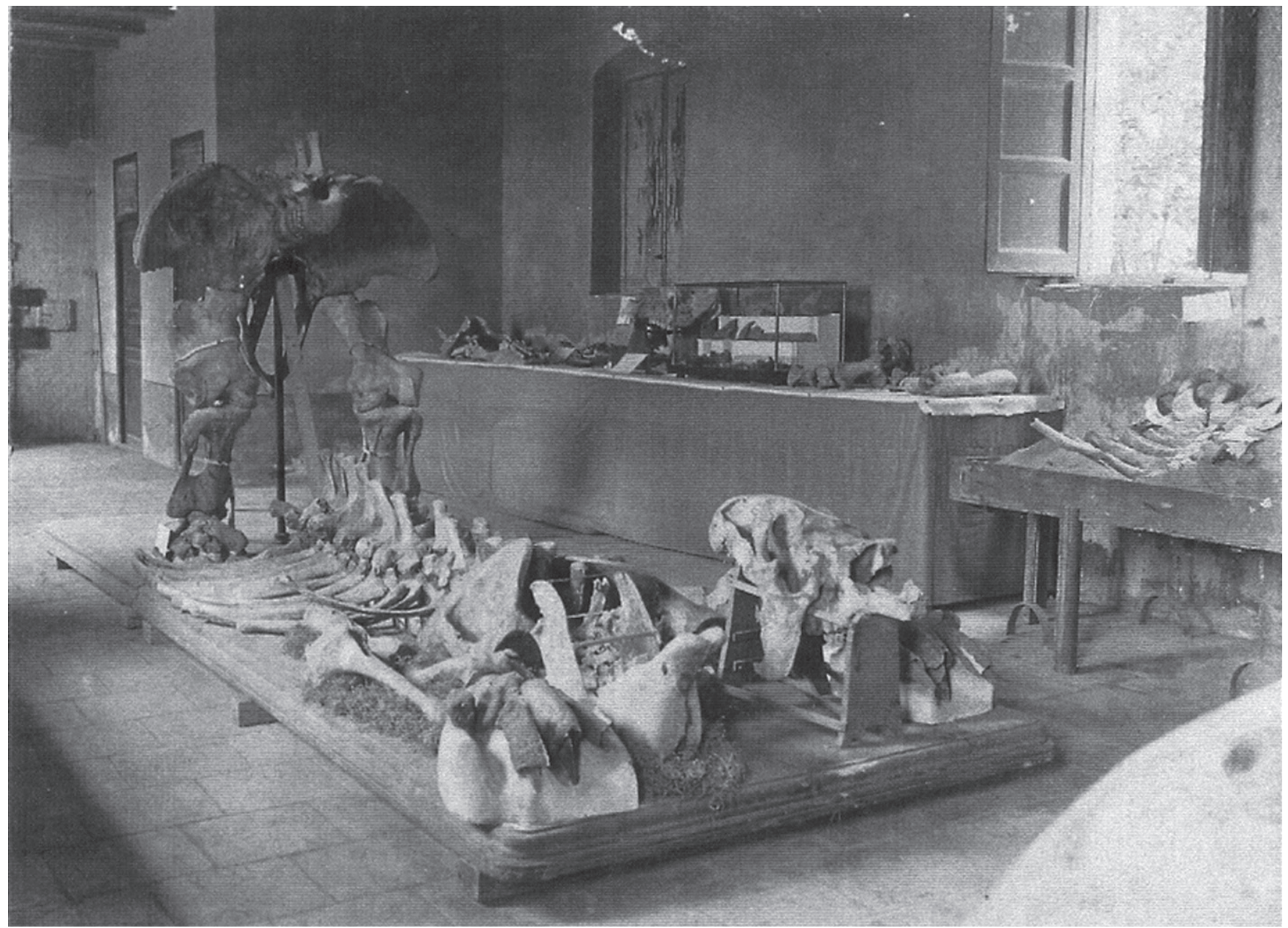

Figura 1. Fotografía del megaterio, ya reconstituido, tomada en el Hospital de San Pablo por Vicente Barberá Masip en octubre de 1902. Esta imagen, cuyo original se encuentra en el Departamento de Zoología de la Facultad de Ciencias Biológicas de la Universitat de València, es la más antigua del megaterio localizada hasta ahora.

The megatherium at the "Hospital de San Pablo", in 1902. This picture, taken by Vicente Barbera Masip is the most ancient we have found. The original belongs to the Zoology Department of the Faculty of Biological Sciencies of the Universitat de València. 


\section{EL MEGATERIO DEL RIO SAMBOROMBÓN}

De las dos referencias a materiales asignados al género Megatherium Cuvier, 1796 que aparecen consignadas en el inventario más antiguo de la colección que se conoce (Carles, 1889), la primera corresponde a un individuo muy completo $-\mathrm{y}$ susceptible de ser montado-, procedente del Lujanense (sensu Cione \& Tonni, 1999) del Rio Samborombón. Los restos del ejemplar llegaron a Valencia desmontados y embalados, y las indicaciones del colector acerca de su buen estado de conservación y de la facilidad con que podía montarse, así como su interés intrínseco, motivaron la decisión de comenzar el montaje de los ejemplares de la colección por éste. Seguramente fue el propio colector quien emprendió los trabajos preliminares, pues en 1890 ya se habían iniciado (Vilanova y Piera, 1890).

Instalada la colección en el Hospital de San Pablo en 1896 (Salinas Jaques, 2001), se reemprendió la reconstitución de los huesos del megaterio y su ordenación (Giner San Antonio, 1906) y, en marzo de 1902, Eduardo Boscá comunicó a la Comisión de Monumentos del Ayuntamiento que el ejemplar estaba en condiciones de exponerse (Salinas Jaques, 2001); en octubre - con motivo de la celebración del IV Centenario de la Fundación de la Universidad de Valencia-, la colección se mostró públicamente (Giner San Antonio, 1906).

Una fotografía de Barberá Masip, conservada en el Departamento de Zoología de la Facultad de Ciencias Biológicas de la Universitat de València, muestra el megaterio tal y como se hallaba entonces: la pelvis y extremidades posteriores pueden verse ya montadas sobre el armazón metálico definitivo, así como el esternón y las costillas esternales, que se encuentran sobre una mesa. El resto del esqueleto, ordenado, descansa sobre un tablero (Fig. 1). Los huesos hioideos del individuo también estaban ya montados entonces (Boscá Casanoves, 1902) y a partir de 1909 , se expusieron, junto con un par de sesamoideos, bajo un fanal de cristal (Boscá Casanoves, 1909).

\section{LAS HIPÓTESIS SOBRE LA ALIMENTACIÓN DEL MEGATERIO DE EDUARDO BOSCÁ}

\section{El estudio del aparato lingual del Megaterio (1902)}

También en 1902, Eduardo Boscá publicó una descripción morfológica y funcional del aparato lingual del megaterio, a partir de la cual ponía en tela de juicio el régimen vegetariano atribuido al animal por otros autores, y cuestionaba la idoneidad de los montajes de esqueletos en los que sus patas delanteras se presentaban apoyadas sobre un tronco de árbol. En opinión de Boscá, la disposición, forma y desgaste de los molares del ejemplar de la colección Rodrigo Botet, y la funcionalidad de su aparato lingual, conducían a la sospecha "de que [los megaterios] capturasen sus alimentos por sorpresa, como lo hace el camaleón, cuyo cuerpo inmóvil contrasta con la rapidez de los movimientos de su lengua para la prensión de los insectos de los que se mantiene" (Boscá Casanoves, 1902).

La publicación de esta original hipótesis sobre la biología del megaterio constituía el punto de partida para estudios posteriores, cuya realización se vería dificultada tanto por la falta de materiales de comparación y la pobreza de recursos bibliográficos, como por la estrechez de miras del Ayuntamiento de Valencia (Archivo de la Junta para la Ampliación de Estudios JAE, Expediente 22-447). Sin embargo, Eduardo Boscá, tan consciente del alcance de estas limitaciones como decidido a superarlo, encontró una fórmula que le permitiría ampliar sus estudios fuera de España.

\section{Los viajes de estudio de Eduardo y Antimo Boscá (1909-1911)}

En septiembre de 1908, apenas dos meses después del traslado de la colección Rodrigo Botet al Almudín, Eduardo Boscá solicitó de la Junta para la Ampliación de Estudios e Investigaciones Científicas (JAE) permiso y cobertura económica para visitar los museos argentinos de La Plata y Nacional de Buenos Aires - hoy Museo Argentino de Ciencias Naturales "Bernardino Rivadavia"-, justificando su petición en la importancia de la colección paleontológica y en el agotamiento de los medios de estudio disponibles en España (Archivo de la Junta de Ampliación de Estudios. JAE. Expediente 22447). La respuesta de la JAE se concretó en la concesión de cuatro pensiones reservadas que permitieron que Eduardo Boscá y su hijo Antimo (Valencia, 1874-1950) visitaran no sólo los museos argentinos, sino también los europeos que contaban entre sus fondos con materiales paleontológicos argentinos.

En relación con el estudio del megaterio, dos eran los objetivos fijados por Eduardo Boscá: confirmar o desmentir la asignación a Megatherium americanum Cuvier, 1796 del individuo del Rio Samborombón y estudiar la flora y la fauna entomológica de las Pampas (ibidem).

El cumplimiento de los objetivos propuestos se materializó en un notable impulso en los trabajos de montaje de los ejemplares de la colección paleontológica a partir de 1912 y en la publicación, bajo la forma de opúsculos, de los grandes grupos de la colección y de sus ejemplares más conspicuos (Salinas Jaques, 2001). Sin embargo, a la muerte de Eduardo Boscá en 1924, el megaterio, inédito, quedaba en el mismo estado de montaje que en 1907 (Figs. 2, 3). 


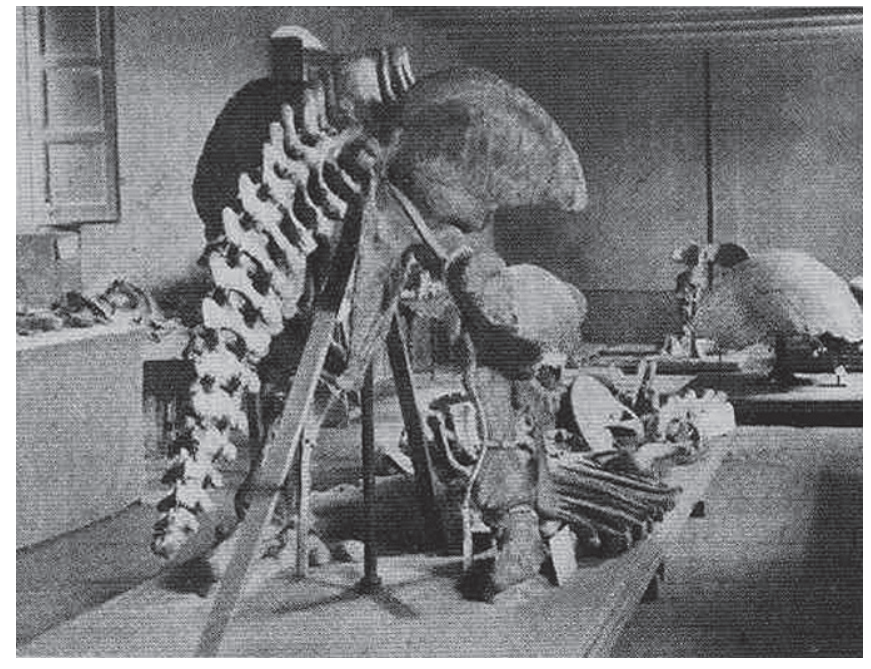

Figura 2. Detalle de una fotografía, de autor desconocido, tomada en el Hospital de San Pablo hacia 1907. El único avance en el montaje del megaterio, según esta imagen, es la adición de la cola (in Barberá, 1907). This is a detail of a picture taken by an anonimous photographer about 1907, at the "Hospital de San Pablo". The skeleton shows his tail locked at place, as the only progress in the state of assembly known in 1902 (in Barberá, 1907).

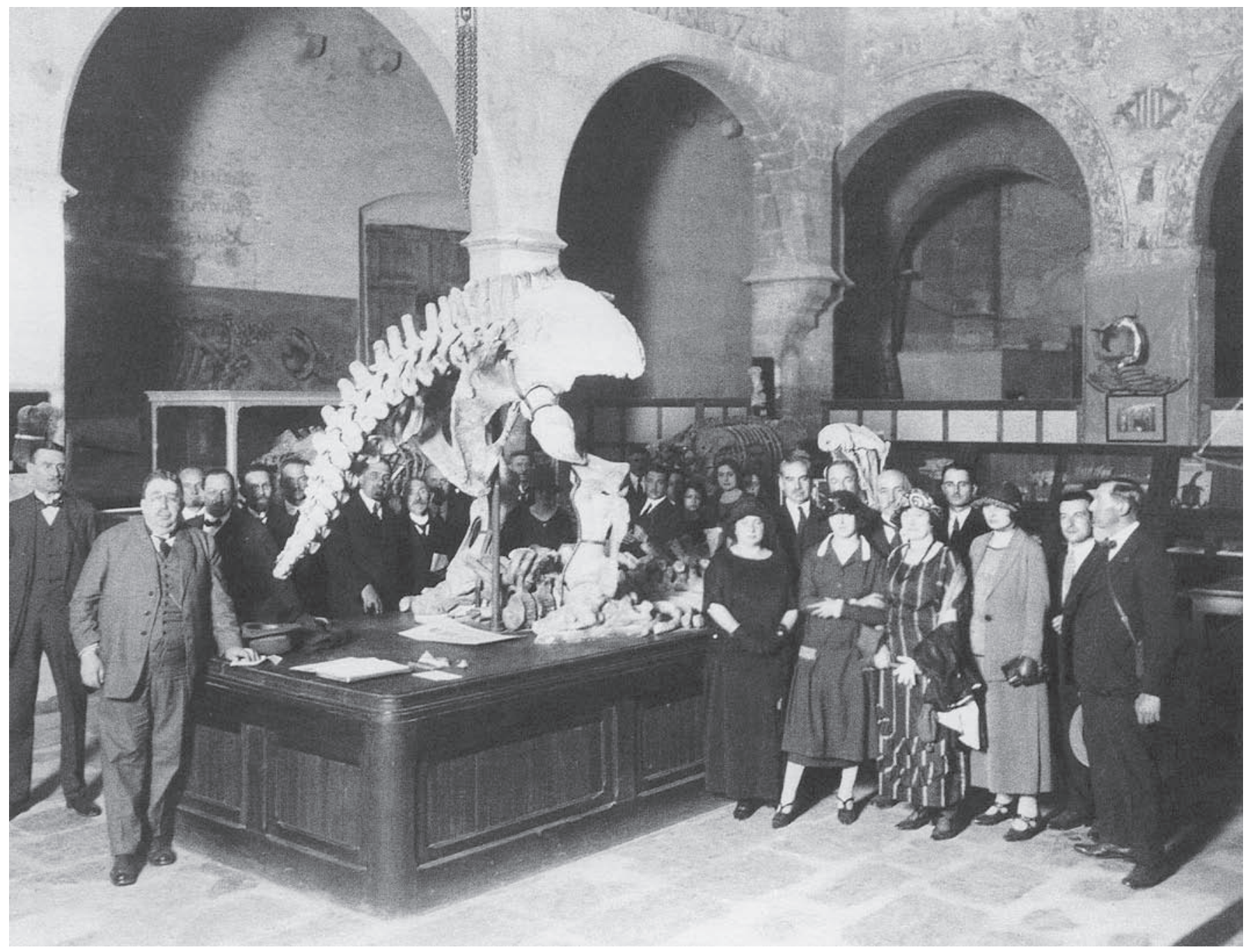

Figura 3. Miembros de la Union International Geodésique et Geophysique fotografiados por Vicente Barberá Masip con motivo de su visita al Museo Paleontológico - ubicado desde 1908 en el Almudín -, el 9 de octubre de 1924. La imagen, que presenta el megaterio tal y como quedó a la muerte de Eduardo Boscá, acaecida cuatro meses antes, no muestra avance alguno en su montaje en relación a la figura anterior (in Aguilar, 1996).

This picture shows the megatherium four months after the Eduardo Boscá's death. The skeleton looks like in 1907, as we can see. Barberá Masip took this picture during a visit to the Almudin of the Union International Geodésique et Geophysique, the $9^{\text {th }}$ October 1924 (in Aguilar, 1996). 


\section{LOS MOTIVOS DE EDUARDO BOSCÁ PARA EL APLAZAMIENTO DEL MONTAJE DEL MEGATERIO}

Un año más tarde, Antimo Boscá reivindicó públicamente la labor de su padre en los siguientes términos:

"[...] el sabio naturalista e inolvidable que fue director de la Colección Botet, doctor E. Boscá, después de más de veinticinco años consagrado al estudio de tan maravillosa Colección, la primera en Europa en su género, hasta última hora dejó para montar el esqueleto del Megaterio, según una nueva concepción, fruto de los estudios y recogidos, con el que estas cuartillas escribe, en los $\mathrm{Mu}$ seos de París y Londres (Cirujanos e Historia Natural), de Buenos Aires y de La Plata, sin olvidar al de Madrid, nueva idea que fue aprobada por los especialistas en la materia" (Boscá Seytre, 1925).

La nota, apologética, constituía la comunicación de que Eduardo Boscá había estudiado el megaterio en profundidad y justificaba - precisamente en lo dilatado y minucioso de sus estudios - la demora de un cuarto de siglo en su montaje. Semejante justificación resulta eufemística, reconstruida la historia de la colección entre 1889 y 1940: bien conocía Antimo que su padre, preocupado por el destino de la colección y desalentado por la desidia municipal, había intentado, a espaldas del Ayuntamiento, promover la transferencia de la responsabilidad sobre la colección Rodrigo Botet al Gobierno Español en dos ocasiones, entre 1908 y 1916, sin éxito (Salinas Jaques, 2001). Además - eso sí fue público y conocido por la municipalidad-, entre 1913 y 1924 se desarrollaron una serie de iniciativas en pos de la institucionalización de la práctica naturalista en Valencia, entre cuyos objetivos se encontraba la ubicación en locales idóneos del Laboratorio de Hidrobiología, un Museo Regional y la propia colección paleontológica y Eduardo Boscá estaba implicado directamente en éstas, como miembro de la Sección de Valencia de la Real Sociedad Española de Historia Natural y como director del Museo Paleontológico (Catalá Gorgues, 1997, 2000; Salinas Jaques, 2001).

El verdadero objetivo del aplazamiento del montaje del megaterio por parte de Eduardo Boscá era franquear el traslado de la colección a un lugar digno, manteniendo la esperanza de que los proyectos aludidos llegaran a buen fin, como veremos a continuación.

\section{El estudio inédito del megaterio}

El resultado de los estudios efectuados sobre el megaterio por Eduardo Boscá y revindicados por su hijo en 1925, se halló en forma de manuscrito, incorporado a un expediente administrativo custodiado en el Archivo Municipal de Valencia. Se trata de un estudio sobre los restos de Megatherium Cuvier, 1796 de la colección y, en detalle, del individuo del Rio Samborombón.
El manuscrito completo (Boscá Casanoves, 1923), consta de un prólogo de diez cuartillas y un texto sobre Megatherium Cuvier, 1796 de quince, y lleva por título: "Catálogo inventario de la Colección paleontológica donada por el patricio José Rodrigo Botet, para que figurara en los museos de la ciudad de Valencia”. El prólogo proclama la mentalidad evolucionista de Eduardo Boscá, quien decidió que fuera el esqueleto de Megatherium americanum Cuvier, 1796 el primer ejemplar que figurara en el catálogo. En el manuscrito, sin duda escrito en 1923 (Salinas Jaques, 2001), se describen con todo detalle las partes naturales del esqueleto y se dan sus medidas. En él Eduardo Boscá defiende la hipótesis de una alimentación de tipo insectívoro y el montaje del esqueleto, en sus propias palabras, en posición de encabritamiento, postura en la que la cola forma un trípode con las patas traseras.

En relación con el aplazamiento del montaje, trascribimos lo que dejó escrito el naturalista: "El esqueleto reconstruido ha quedado sin montar, primero por si se consiguiera vaciarlo [reproducir sus elementos óseos en alabastro] por los fines de propaganda [divulgación o difusión] y facilidad de su estudio, y segundo: en espera de local más capaz y adecuado que el Almudín en ruinas." En otro manuscrito, en este caso del Archivo del Museo Palentológico Rodrigo Botet, que nos ha llegado incompleto, fue más sincero: "El trabajo puede darse por terminado, pero aunque la pelvis con la cola y miembros posteriores están montados sobre una gran mesa apropiada, no debe terminarse su montura, pues sería motivo quizá de no trasladar la colección a un edificio más apropiado y decoroso cual lo merece, aparte de ser ya incapaz el local, y de su estado ruinoso lleno de goteras"(Archivo del Museo Paleontológico Rodrigo Botet. AMPRB S/C.).

En relación con el modo de vida del megaterio y de cómo plasmarlo en su montaje, como se ha indicado a propósito de sus estudios del aparato lingual, el naturalista sostenía que el régimen alimenticio del megaterio era entomófago y no folívoro como sugerían otros autores. Para éstos, la conformación de las manos del megaterio y su posibilidad de mantenerse erguido sobre patas traseras y cola, le permitirían acceder a las ramas de los árboles de cuyas hojas se alimentaba. Por eso, muchos de ellos aparecían, como los de París y Londres, montados con las manos apoyadas sobre un tronco de árbol.

Eduardo Boscá - que daba la razón a quienes vieron en el megaterio un gigantesco oso hormiguero - estimaba más idóneo un montaje en el que las manos del esqueleto se apoyaran sobre uno de los enormes nidos columnares (tocurús o tacurús) que construyen las hormigas blancas, de los que la Pampa se encontraba plagada. Al naturalista le parecía muy improbable que los megaterios pudieran alimentarse de hojas de árbol en un lugar donde no había rastro de vegetación arbórea en el registro fósil (Boscá Casanoves, 1902). Sus hipótesis compatibilizaban diver- 
sos aspectos anatómicos y funcionales del esqueleto con la supervivencia en el medio que debió ser la Pampa que habitaron. Así, los grandes soportes que presentan las falanges ungueales de los dedos de las manos, indicarían que el megaterio estaba provisto de fuertes uñas, adecuadas para la destrucción de los nidos de hormigas. Tras atacar la colonia, "las hormigas se derramaban particularmente en torno de su larga lengua vermiforme a la que acometían, mientras el animal, gracias al hioides cuyo complemento era una pieza elástica a cada lado [...], retiraba dicha lengua cuantas veces la sentía llena. La excepcional forma y gran peso (14 kilogramos) de la mandíbula inferior del Megaterio, da la sospecha de una función especial, cual podía ser la permanencia de la boca abierta de modo pasivo, mientras se repetían las cargas de los blandos animales que de tiempo en tiempo eran chafados por las muelas adecuadas cuyas aristas se presentan integras, a pesar de la edad del animal. La apófisis descendente malar, por su longitud y anchura, quitaba la posibilidad de un esguince, fácil en otro caso. En apoyo de que animal tan grande se alimentara de presa pequeña, recordemos las actuales ballenas con respecto al Clione pallas, supliendo la cantidad a la calidad como base alimenticia" (Boscá Casanoves, 1923).

Estas notas constituyen, pues, la concreción de las hipótesis de Boscá sobre el régimen entomófago del megaterio formuladas en 1902 .

Tres años después, Royo y Gómez apoyó las hipótesis sobre la alimentación del megaterio defendidas por Boscá y discutidas por Cabrera en 1926 (Royo y Gómez, 1929). Royo, conocedor del trabajo sobre el aparato hioideo de Boscá, desconocía, sin embargo, las reflexiones recogidas en su estudio manuscrito, lo que queda patente cuando, hablando del trabajo publicado por Sanielevici en 1926 -en el que éste defiende y discute la hipótesis de una alimentación basada en coleópteros xilófagos-, hace hincapié en la novedad aportada por este autor al estudiar las modificaciones sufridas por el aparato masticador en función del tipo de alimentación basándose, no sólo en la forma de los dientes y su disposición, sino "sobre todo en la forma de los huesos como resultado del mayor o menor desarrollo de las inserciones musculares y por lo tanto del modo como han sido tratados los alimentos al llegar a la boca" (Royo y Gómez, 1929). La interpretación que hace Sanielevici (1926) del desplazamiento hacia los molares del procesus descendens es diferente de la que hace Eduardo Boscá, que, como hemos visto en la transcripción de su manuscrito, tuvo muy en cuenta.

\section{LA CULMINACIÓN DEL MONTAJE DEL MEGATERIO}

Según la perspectiva de Sanielevici, un montaje del megaterio apoyado sobre un tronco arbóreo sería idóneo, tal y como debió considerarlo, conocedor o no de este trabajo, Francisco Beltrán, a juzgar por la forma en que dejó montado el ejemplar: con las manos apoyadas sobre un tronco de eucalipto.

Francisco Beltrán, ávido de acaparar cargos y honores y completamente desinteresado tanto del estudio de las colecciones del museo que dirigía, como de su destino (Catalá Gorgues, 2000; Salinas Jaques, 2001), se apresuró a hacer suyos ante el Ayuntamiento los trabajos emprendidos por Eduardo Boscá y el montador Carlos Maicas

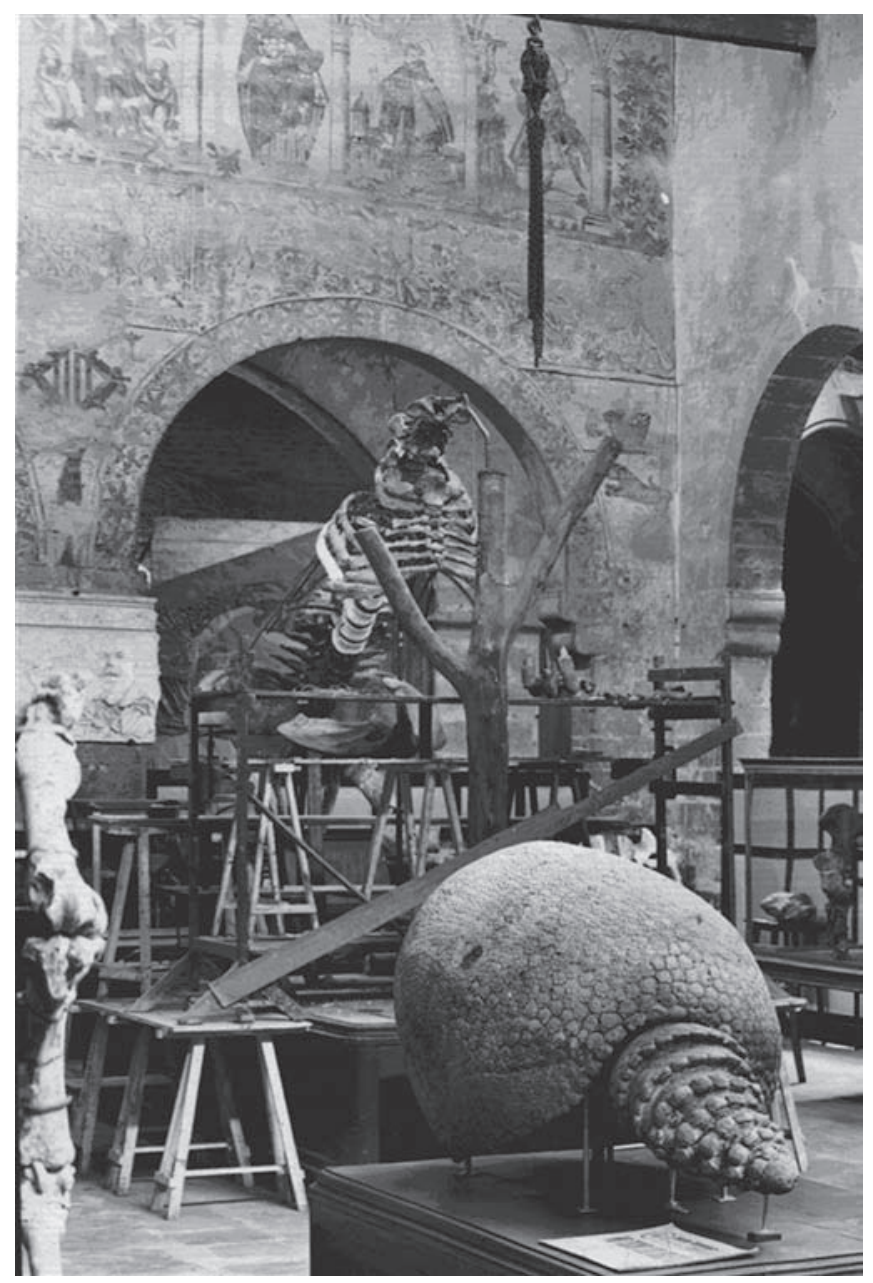

Figura 4. Detalle de una panorámica del Almudín tomada por Barberá Masip en 1927, en la que puede verse el cambio de orientación del esqueleto, ya prácticamente ultimado en su montaje, así como el tronco de eucalipto sobre el que habrían de apoyarse las manos del megaterio, según la concepción de Francisco Beltrán (in Sabaté, 1996).

This is a detail of a picture taken by Barberá Masip in 1927 at the "Almudin". The picture shows the megatherium few months before finishing his assembly. Here, we can see a change in the skeleton position and too, the eucaliptus tree where Beltrán though to lest on the forelimbs of the animal (in Sabaté, 1996). 
$\mathrm{y}$, aunque no cabe la menor duda de que el montaje del megaterio se completó bajo su dirección, todo parece indicar que los trabajos - desarrollados entre 1926 y enero de 1928-se redujeron a realizar algún retoque en ciertos elementos óseos, terminar la construcción del armazón de sustentación, completar el ensamblaje del esqueleto (Fig.4) y enviar a la Subcomisión de Monumentos larguísimos y falseados informes de actividad, convenientemente orientados en provecho de su imagen ante el Ayuntamiento de Valencia, como se desprende del cotejado de estos escritos con los debidos a Eduardo y Antimo Boscá, fechados entre 1902 y 1925, y con los libros de gastos que se conservan en el Archivo del Museo Paleontológico Rodrigo Botet.

Tal vez pueda calificarse de afortunada la circunstancia de que fuera José Cebolla y no Carlos Maicas - montador de la colección entre mediados de 1890 y 1921 (Salinas Jaques, 2001)- quien se encargara de terminar el montaje del megaterio. Apenas diez años después, Cebolla fue responsable, hallándose Beltrán en paradero desconocido, de la evacuación de la mayor parte de la colección paleontológica para su protección en los sótanos del Banco de Aragón durante la Guerra Civil (Salinas Jaques, 1999). El megaterio, como el resto de los ejemplares trasladados, se devolvió al Almudín en mayo de 1939 y fue montado de nuevo con arreglo a como lo había sido en 1927 (Salinas Jaques, 2001).

Entre febrero de 1990 y mayo de 1999, se desmontó y volvió a montar el megaterio en dos ocasiones: la primera con motivo de la evacuación del Almudín y el montaje del Museo Paleontológico en los bajos de la Casa Consistorial (Fig. 5) (ver Belinchón et al., 1992) y la segunda, con motivo de la creación del Museo Municipal de Ciencias Naturales (ver Sánchez Ferris et al., 1999).

Un siglo después de que Eduardo Boscá cuestionara los hábitos alimenticios del megaterio admitidos tradicionalmente, algunos autores modernos ponen en tela de juicio los nichos ecológicos que se han venido adjudicando a los grandes mamíferos del Lujanense (ver Fariña, 1996). Es en este marco conceptual que se han formulado nuevas hipótesis acerca del modo de vida del megaterio que cuestionan, cuando no rechazan, una alimentación vegetariana para este animal (ver Fariña \& Blanco, 1996).

\section{AGRADECIMIENTOS}

Agradezco sinceramente su aliento en la gestación de esta comunicación a las XIX Jornadas de Paleontología, a los Doctores Julio Gómez-Alba, conservador del Museu de Geologia de Barcelona y Francisco Pelayo, Científico Titular del CSIC. Al Dr. Richard Fariña, profesor de la Facultad de Ciencias de Montevideo, debo agradecerle sus entusiastas comentarios y sus interesantes apostillas al manuscrito en su calidad de revisor. Para terminar, agradezco vivamente al Dr. Rodolfo Gozalo del Departamento de Geología de la Universitat de València, la entrega y cariño con que oficia como Editor de esta revista.

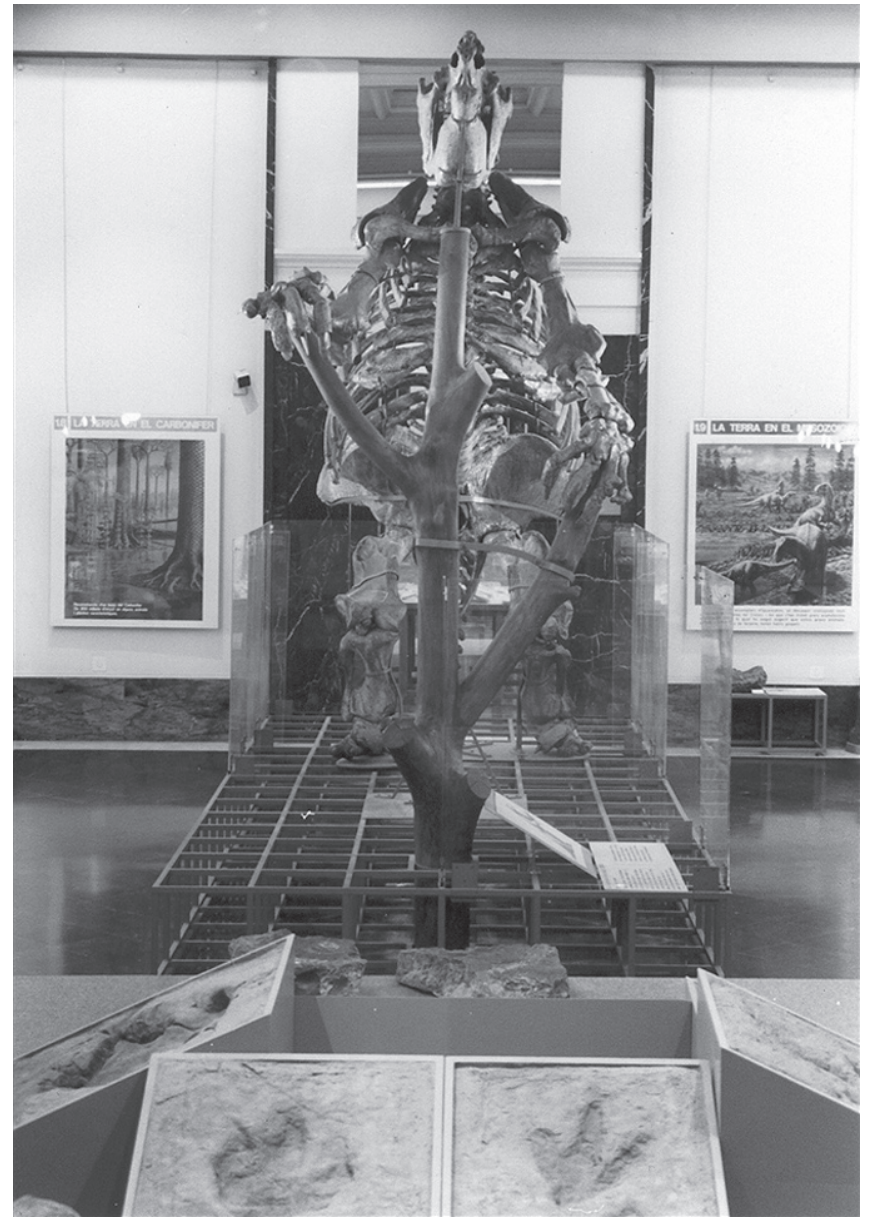

Figura 5. El megaterio en la Sala Municipal de Exposiciones (1991-1999). Este montaje reproduce el realizado bajo la dirección de Beltrán, el mismo que puede verse actualmente en el Museo Municipal de Ciencias Naturales. Fotografía de autor desconocido. Archivo Fotográfico del Museo Paleontológico, Parte Moderna. AFMPM 109.14.

The Rodrigo Botet Museum moved from the "Almudin" to he "Sala Municipal de Exposiciones" in 1990. The megatherium was taken the pieces and assembled as it was in 1927. Then, in 1999, this process was repeated and, today, we can see at the "Museo Municipal de Ciencias Naturales" the megatherium as it is shown at this picture, taken by an unknown photographer in 1991. Archivo Fotográfico del Museo Palentológico, parte Moderna. AFMPM 109.14.

\section{BIBLIOGRAFÍA}

Aguilar, I. 1996. Arte, cultura y sociedad. In: El Almudín de Valencia (Dir. A. Llopis). FCC Medio Ambiente S.A., Valencia, 73-108.

Alberdi, M. T., Leone, G. \& Tonni, E. P. 1995. Taxonomía paleontológica. In: Evolución biológica y climática de la región pampeana durante los cinco últimos millones de años. Un ensayo de correlación con el Mediterráneo Oc- 
cidental. Consejo Superior de Investigaciones Científicas, Madrid, 365-380.

Barberá Martí, F. 1907. Nota relativa al esqueleto humano de la colección paleontológica Botet en Valencia. In: Linneo en España. Homenaje a Linneo en su segundo centenario 1707-1907. Mariano Escar, Zaragoza, 505-516.

Belinchón, M, Carrió, V., Faura, M., García-Forner, A., Montoya, P., Peñalver, E. \& Salinas Jaques, A. 1992. El Museo Paleontológico de Valencia. Proceso de traslado y perspectivas de futuro. In: Paleontología y Sociedad. Sociedad Española de Paleontología y Universidad de Granada, Granada, 51-58.

Boscá Casanoves, E. 1899. Noticias sobre una colección paleontológica regalada al Excmo. Ayuntamiento de Valencia. Anales de la Sociedad Española de Historia Natural (Actas), 28, 82-90.

Boscá Casanoves, E. 1902. Notas sobre un Megaterio existente en Valencia. Boletín de la Real Sociedad Española de Historia Natural, 2, 139-146.

Boscá Casanoves, E. 1909. Catálogo Guía de la colección paleontológica de J. Rodrigo Botet, dedicado al Congreso de Valencia de la Asociación Española para el Progreso de las Ciencias. Sucesores de Emilio Pascual, Valencia, $16 \mathrm{pp}$.

Boscá Casanoves, E. 1923. Catálogo inventario de la Colección paleontológica donada por el patricio José Rodrigo Botet, para que figurara en los museos de la ciudad de Valencia (manuscrito inédito). Archivo Municipal de Valencia. AMV. Expedientes de la Comisión de Monumentos. Año 1923. Expte. 26/1923, 26pp.

Boscá Seytre, A. 1925. El Megaterio Sud-Americano. Las Provincias, 18-02-1925.

Cabrera, A. 1926. Sobre la alimentación del megaterio. Boletín de la Real Sociedad Española de Historia Natural, 26, 388-391.

Carles, E. de. 1889. Catálogo de la Colección paleontológica que el Ingeniero Don José Rodrigo Botet, regala a la Ciudad de Valencia, obtenida en las exploraciones efectuadas en la provincia de Buenos Aires (República Argentina) por el naturalista D. Enrique de Carles (manuscrito inédito). Archivo del Museo Palentológico Rodrigo Botet, AMPRB S/C.

Catalá Gorgues, J. I. 1997. El Museu Regional i el Palau de les Ciències Naturals: Iniciatives d'institucionalització de la història natural a la València del primer terç del segle XX. In: Actes de las IV Trobades d'Història de la Ciencia i de la Técnica. Societat Catalana d'Història de la Ciència i de la Tècnica, Barcelona, 119-126.

Catalá Gorgues, J.I. 2000. Los cultivadores de la Historia Natural en Valencia (1909-1940). Tesis doctoral. Universitat de València, 934 pp. (inédita).
Cione, A. L. \& Tonni, E. P. 1999. Biostratigraphy and chronological scale of upper-most Cenozoic in the Pampean Area, Argentina. In: Quaternary vertebrate paelontology in South America (eds. E. P. Tonni \& L. A. Cione). Quaternary of South America and Antartic Peninsula, Special volume 12, 23-51.

Fariña, R. A. 1996. Trophic relationships among Lujanian mammals. Evolutionary Theory, 11, 125-134.

Fariña, R. A. \& Blanco, R. E. 1996. Megatherium, the stabber. Proceedings of the Royal Society of London B, 263, 1725-1729

Giner San Antonio, M. 1906. Exposición paleontológica. In: Crónica del IV centenario de la Universidad de Valencia. (rec. Giner San Antonio, M.), Doménech, Valencia, 5 pp.

Royo y Gómez, J. 1929. Sobre biología del megaterio. Extracto de las conferencias y reseñas científicas de la Real Sociedad Española de Historia Natural, 4, 31-35.

Sabaté, I. 1996. Restauración de las pinturas murales. In: $E l$ Almudín de Valencia (dir. A. Llopis). FCC Medio Ambiente, S.A., Valencia, 129-147.

Salinas Jaques, M. A. 1999. Medidas para la protección de los fondos del Museo Paleontológico adoptadas por el Consejo Municipal de Valencia durante la Guerra Civil Española (1936-1939). In: Actas de las XV Jornadas de Paleontología (ed. I. Rábano). Instituto Tecnológico Geominero de España, Ministerio de Medio Ambiente, Madrid, 2, 439-441.

Salinas Jaques, M. A. 2001. Las colecciones paleontológica y conquiológica del Museo Paleontológico J. Rodrigo Botet de Valencia: inventario faunístico, importancia científica, museística e histórica. Tesis doctoral. Universitat de València, 803 pp. (inédita).

Sánchez Ferris, E.J., Santamaría, R., Montoya, P., Momparler, R., Cañizares, A., Barcelón, G., Gosálvez, P., Guillem, J., Marín, M. D., y Dolz, A. 1999. Movilización y restauración de fondos paleontológicos para su instalación en el nuevo Museo de Ciencias Naturales de Valencia. In: Actas de las XV Jornadas de Paleontología (ed. I. Rábano). Instituto Tecnológico Geominero de España, Ministerio de Medio Ambiente, Madrid, 2, 442-446.

Sanielevici, H. 1926. La vie des mammifères fossiles déchiffrée á l'aide de l'anatomie et de la physiologie comparées de l'appareil masticateur. Bulletin de la Societé Roumaine des Sciencies, Bucarest, 660 pp.

Vilanova y Piera, J. 1890. Noticias sobre el Museo paleontológico del señor Botet de Valencia. Actas de la Real Sociedad Española de Historia Natural, 19, 19-25.

Manuscrito recibido: 1 de Diciembre, 2003 Manuscrito aceptado: 4 de Marzo, 2005 